\title{
Chemical Oscillation with Periodic Adsorption and Desorption of Surfactant Ions at a Water/Nitrobenzene Interface
}

\author{
Yasuhiro IKezoe, ${ }^{* 1 \dagger}$ Sadahiro IshIZAKI, ${ }^{* 1}$ Hiroharu YuI, $* 1, * 2$ Masanori FuJINAMI $,{ }^{*} 1, * 3$ and \\ Tsuguo SAWADA $* 2, * 4$
}

*1 Department of Advanced Materials Science, Graduate School of Frontier Sciences, The University of Tokyo, 5-1-5 Kashiwanoha, Kashiwa, Chiba 277-8561, Japan

*2 Core Research for Evolutional Science and Technology (CREST), Japan Science and Technology Agency (JST), 5-1-5 Kashiwanoha, Kashiwa, Chiba 277-8561, Japan

*3 Department of Applied Chemistry \& Biotechnology, Chiba University, 1-33 Yayoi, Inage, Chiba 263-8522, Japan

*4 Department of Chemical Engineering, Tokyo University of Agriculture and Technology,

2-24-16 Naka-cho, Koganei-city, Tokyo 184-8588, Japan

\begin{abstract}
Chemical oscillations with periodic adsorption and desorption of surfactant ions, alkyl sulfate ions, at a water/nitrobenzene interface have been investigated. The interfacial tension was measured with a quasi elastic laser scattering (QELS) method and the interfacial electrical potential was obtained. We found that this oscillation consists of a series of abrupt adsorptions of ions, followed by a gradual desorption. In addition, we observed that each abrupt adsorption was always accompanied by a small waving motion of the liquid interface. From the analysis of the video images of the liquid interface or bulk phase, we could conclude that each abrupt adsorption is caused by nonlinear amplification of mass transfer of ions from the bulk phase to the liquid interface by a Marangoni convection, which was generated due to local adsorption of the surfactant ions at the liquid interface that resulted in the heterogeneity of the interfacial tension. In the present paper, we describe the mechanism of the chemical oscillation in terms of the hydrodynamic effect on the ion adsorption processes, and we also show the interfacial chemical reaction with ion exchange during the ion desorption process.
\end{abstract}

(Received August 10, 2004; Accepted September 8, 2004)

\begin{tabular}{llll}
\hline 1 Introduction & 1509 & $3 \cdot 3$ Ion exchange reaction & \\
2 Experimental & 1510 & 4 Conclusion & 1513 \\
3 Results and Discussion & 1511 & 5 Acknowledgements & 1514 \\
$3 \cdot 1$ Chemical oscillation & & 6 References & 1514 \\
$3 \cdot 2$ Marangoni convection & & & \\
\hline
\end{tabular}

\section{Introduction}

An interface formed by two immiscible liquids is a specific area where chemical composition and physical properties steeply change on the nano-meter scale. ${ }^{1}$ Some characteristic phenomena are found: for example, adsorption and desorption of molecules, molecular recognition, ${ }^{2,3}$ solvent extraction. ${ }^{4}$ Chemical oscillations at a liquid/liquid interface have also been drawing attention from researchers in terms of not only the nonlinear chemistry but also for studies of biomimetic system. Since Dupeyrat and Nakache ${ }^{5}$ first reported the electrochemical potential oscillation at the water/nitrobenzene interface in the 1970 's, many kinds of chemical oscillation systems, not only water/oil two-phase systems ${ }^{5-11}$ but also water/oil/water three-

† To whom correspondence should be addressed.

E-mail: ikezoe@laser.t.u-tokyo.ac.jp phase systems ${ }^{12-28}$ or gas/liquid interfaces, ${ }^{29-32}$ have been developed and extensively investigated.

Recently, we found a new-type interfacial oscillatory phenomenon with periodic adsorption and desorption of sodium alkyl sulfate at a water/nitrobenzene interface. ${ }^{23-26}$ As previously reported in other interfacial oscillations, our system also shows an oscillation of electrical potential between the aqueous phase and the oil. The electrical potential is generated by unbalanced distribution of charged elements, which is not a simple phenomenon because such a situation is caused by adsorption of ions at the liquid/liquid interface, differences of concentration of the ions between the two liquid phases, and differences of ion mobility across the interface. As a result, understanding the chemical oscillation only from an electrical potential measurement is quite difficult. That is why, while most experiments showed a periodic oscillation pattern of electrical potential as a common property, the mechanisms proposed were not always similar. 


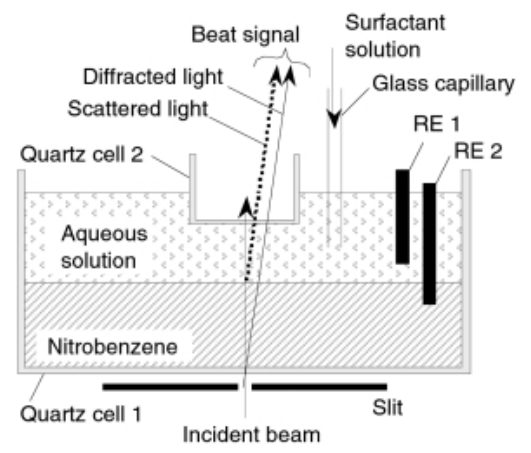

Fig. 1 Schematic figure of the experimental setup for the measurement of interfacial tension and electrical potential at the water/nitrobenzene interface. The interfacial tension was measured by the QELS method.

These mechanisms can be classified into two main groups: one explanation is that the oscillation includes the rapid adsorption of molecules or ions followed by gradual desorption, ${ }^{29-32}$ and the other is the opposite. ${ }^{6-8,10,12,17}$ In addition, Maeda et al. ${ }^{13,14}$ gave a novel explanation focusing on the generation and reduction of the maximum wave in the ion transfer polarograms. The maximum wave is also caused by the interfacial instability due to the adsorption of the ion at the interface. In all cases, the chemical oscillation at the liquid/liquid interface is closely related to the adsorption and desorption of the surfactant ions. Therefore it is important to know the kinetics of the ion adsorption and desorption. Interfacial tension change with the density of the surfactant ions adsorbed at the interface, that is to say, the larger the density is, the smaller the interfacial tension. And hence, we have developed an instrument for the simultaneous measurement of the interfacial electrical potential and the interfacial tension.

For about ten years, we have developed a time-resolved Quasi Elastic Laser Scattering (QELS) method to measure a time course of interfacial tension with adsorption or desorption of surfactant molecules at the interface. ${ }^{33}$ The interfacial tension can be calculated from the frequency of the light scattered by a thermally generated capillary wave at the interface. The most popular form of interfacial tensiometry for investigating the chemical oscillation is a Wilhelmy plate method. $5,8,10,11,20,22,29-32$ One can measure the interfacial tension by directly measuring the force acting on a small glass or platinum plate in contact with the interface. However, such a contact probe physically disturbs the interface and might change the dynamic behavior of molecules at the interface. In fact, there have been some reports claiming that the hydrophilicity of the inner wall of the experimental cell influenced not only the oscillation pattern, but also whether it appears or not. According to the report by Shioi et al. ${ }^{20}$ the oscillatory interfacial tension derived from the Wilhelmy plate tensiometry does not reflect the oscillatory reaction at the interface, but rather the movement of the interface. Furthermore, the oscillatory phenomena were generally considered to include two-dimensionally inhomogeneous reactions and diffusion kinetics. Therefore, such a contact type surface tensiometry is not appropriate for studying the chemical oscillation. From the same reason, a dynamic drop volume method, which is quite useful for the analysis of ordinary adsorption dynamics at the interface, is not appropriate for the analysis of the chemical oscillation, either. Thus, since the QELS method allows noncontact and nonperturbative measurements of the dynamic interfacial tension, we employed it for studying the chemical oscillation system.

A well-known nonlinear chemical reaction is the BelousovZhabotinsky reaction, which consists of a periodic redox reaction in a bulk solution and has been understood by the autocatalytic chemical reaction scheme. On the other hand, the chemical oscillation at the interface is not understood simply by a chemical reaction scheme at the interface; the chemical reaction such as adsorption or desorption of molecules occurred at the interface, and mass-transfer occurred both in the bulk phase and in the interface. It is noteworthy that most chemical oscillations at a liquid/liquid interface were accompanied by a movement of liquid at the interface or in the bulk. However, the movement was simply explained as a kind of instability of interface and was not discussed in detail. As far as we know, precise discussions on the hydrodynamic effect have been limited to some reports. ${ }^{16,20,29-32}$ Since the movement of liquid can cause a flow of liquid near the interface and can substantially change the mass transfer, it is important to understand how the instability at the interface was generated and how the movement of the interface occurred. Therefore, we also focused on the movement of the interface in the chemical oscillation system as well as the adsorption and desorption of the surfactant ions; we analyzed the dynamic behavior of the liquid under the chemical oscillation with the aid of the video images taken by a CCD camera.

In the present paper, we first introduce newly found chemical oscillations at sodium alkyl sulfates and we describe the mechanism of them from the viewpoint of hydrodynamic effects on the chemical reaction and mass transfer. And next, we also discuss the chemical reaction with ion exchange at the interface.

\section{Experimental}

Figure 1 is a schematic diagram of the experimental setup for the QELS and an interfacial electrical potential measurement. A brief outline of the principle of the QELS method follows. An incident laser beam normal to the liquid/liquid interface was scattered quasi-elastically with a Doppler shift by a capillary wave, which was generated due to thermal fluctuations. The scattered beam was optically mixed with a local beam produced by a slit to obtain an optical beat in the mixed light. The obtained beat frequency corresponds to the capillary wave frequency, which is approximately expressed by Lamb's equation based on the theory of hydrodynamics, ${ }^{34}$

$$
f=\frac{1}{2 \pi}\left(\frac{\gamma}{\rho_{\mathrm{w}}+\rho_{\mathrm{o}}}\right)^{\frac{1}{2}} k^{\frac{3}{2}},
$$

where $\gamma$ is the interfacial tension at the water/oil interface, $\rho_{\mathrm{w}}$ is the density of the water phase, $\rho_{\mathrm{o}}$ is the density of the oil phase, and $k$ is the wave number of the capillary wave. The first experiment of QELS method was demonstrated by Kathyl and Ingard in 1967. ${ }^{35}$ Since then, with the improvement of instruments and measurement techniques, this method has been used to study the properties of surfaces of liquid $^{36}$ or liquid crystal, ${ }^{37}$ monolayer, ${ }^{38,39}$ and liquid/liquid interface. ${ }^{40}$ Most experiments were executed at an equilibrium state, while we have succeeded in observing the dynamic behavior of molecules at a liquid/liquid interface. As reported in our previous papers, some novel dynamic behavior of molecules or micelles have been found: for instance, phase transfer catalysis systems across a nitrobenzene/water interface, ${ }^{41}$ anomalous interfacial tension change by AOT micro-emulsion collapses at a heptane/water 


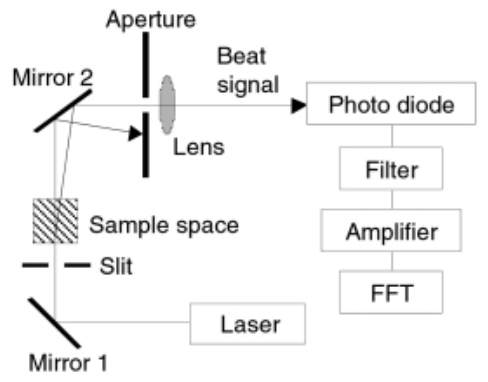

Fig. 2 Block diagram for the QELS measurement. Only an optical beat signal is selected by an aperture in front of a photodiode.

interface, ${ }^{42}$ and enzymatic reaction of phospholipid membranes at a tetradecane/water interface, ${ }^{43}$.

A liquid/liquid interface was formed by gently pouring $10 \mathrm{ml}$ of an aqueous solution of $0.1 \mathrm{M} \mathrm{LiCl}$ onto $10 \mathrm{ml}$ of a nitrobenzene solution of $0.1 \mathrm{M}$ tetra- $n$-butylammoniumtetraphenylborate (TBATPB) in a cylindrical glass cell with an inner diameter of $36 \mathrm{~mm}$ (quartz cell 1) with an optical flat bottom. These electrolytes, $\mathrm{LiCl}$ and TBATPB, were used as supporting electrolytes to measure the electrical potential $(E)$ of the water phase against the NB phase. The electrical potential was measured with a potentiometer using $\mathrm{Ag} / \mathrm{AgCl}$ electrodes (RE1 and RE2) equipped with salt bridges containing an $80 \%$ saturated aqueous solution of $\mathrm{KCl}$ and an aqueous solution of $0.01 \mathrm{M}$ tetra- $n$-butylammonium-chloride (TBACl), respectively. A $10 \mathrm{mM}$ sodium-alkyl-sulfate aqueous solution, which also contained $0.1 \mathrm{M} \mathrm{LiCl}$ as in the aqueous phase, was injected through a glass capillary with an inner diameter of $0.3 \mathrm{~mm}$ into the aqueous phase at a constant rate with a syringe pump (LMS Model 210). A small quartz cell (quartz cell 2) was used in order to prevent the transmitted light scattered at the liquid/air interface from entering the photodiode.

Figure 2 is a block diagram of the experimental system. The beam from a YAG laser (CrystaLaser, Model GCS-200s, 532 $\mathrm{nm}, 60 \mathrm{~mW}$ ) passes through a $0.3 \mathrm{~mm}$ slit below quartz cell 1 . Only an optical beat signal is selected by an aperture in front of an avalanche photodiode (Hamamatsu Photonics, C5331-11) In the present study, the diffracted light of the sixth order was selected. The angle between the transmitted light and diffracted light was calculated numerically from the equation of the Fraunhofer diffraction; the wavelength of the capillary wave observed was found to be $35 \mu \mathrm{m}$. Signals from the photodiode pass through an isolation amplifier and a high-pass filter and are FFT-analyzed by a real-time spectrum analyzer (Sony Tektronix, Model 3056). The interfacial tension and interfacial potential have been simultaneously measured while the surfactant solution has been injected into the aqueous phase.

We used a CCD camera (ELMO, MN43H) for monitoring the movement of the liquid/liquid interface or bulk liquid; the movement was investigated by analyzing the movement of a small amount of carbon powder scattered on the interface. The water used for preparing the aqueous solution was purified by a Millipore Milli-Q System. The materials used were prepared as shown in Ref. 29.

\section{Results and Discussion}

\section{3·1 Chemical oscillation}

Figure 3 shows typical oscillation behavior of interfacial tension and electrical potential. ${ }^{25}$ In this experiment, the tip of

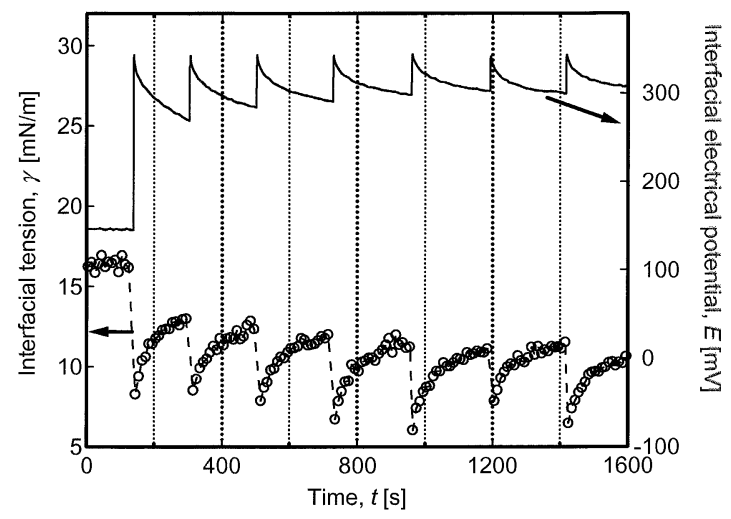

Fig. 3 Time course of the interfacial tension and interfacial electrical potential for the chemical oscillation with sodium dodecyl sulfate..$^{25}$

the capillary was located $8 \mathrm{~mm}$ above the liquid/liquid interface, and the injection rate of the sodium dodecyl sulfate (SDS) aqueous solution was $3 \mu \mathrm{l} / \mathrm{min}$. Open circles and solid lines show the interfacial tension $(\gamma)$ and electrical potential $(E)$, respectively. In the graph, time zero means the time at which the SDS solution injection was started. For about $2 \mathrm{~min}$ (induction period) after injection of the SDS solution into the water phase, no notable changes in $\gamma$ and $E$ were observed. There was a slow convection as a result of the injection of the SDS. In addition, convection was also caused by the difference in the density of the SDS solution, which is slightly heavier than the bulk phase. Therefore, the induction period is considered to be determined mainly by the time for the $\mathrm{DS}^{-}$ions to reach the interface. After the induction period, an abrupt decrease of $\gamma$ and an increase of $E$ appeared simultaneously. Furthermore, just after the oscillation, $\gamma$ and $E$ gradually increased and decreased, respectively. These two processes periodically occurred every 3 to $4 \mathrm{~min}$, and the amplitude gradually reduced. In that figure, although the time course of $\gamma$ and $E$ is shown only for the periods of $1600 \mathrm{~s}$ (corresponding to the $80 \mu \mathrm{l}$ injection of SDS solution), oscillation processes indeed continued until the injection of SDS solution was stopped (1000 $\mu$ l injection).

As the decrease of $\gamma$ should correspond to adsorption of surface-active molecules, the abrupt decrease of the interfacial tension in this experiment could be explained by a rapid adsorption of DS- ions at the liquid/liquid interface. In addition, a rapid increase of $E$ could be explained by the formation of an interfacial electrical double layer by $\mathrm{DS}^{-}$ions and their counterions at the interface.

\subsection{Marangoni convection}

Hereafter, we use the following abbreviation, $\mathrm{SC}_{\mathrm{n}} \mathrm{S}$, for each sodium alkyl sulfate, $\mathrm{C}_{n} \mathrm{H}_{2 n+1} \mathrm{SO}_{4} \mathrm{Na}(n=6,8,10,12$, and 14$)$, respectively. When we performed experiments similar to the above with $\mathrm{SC}_{\mathrm{n}} \mathrm{S}$, we could also observe oscillation phenomenon in each material. Although they were quantitatively different from each other, these results indicate that an oscillatory phenomenon is not a specific feature of some few materials.

The abrupt adsorption at the oscillation was always accompanied by a small waving motion of the liquid/liquid interface. At the same time, we could also see a tangential flow along the interface from the area below the outlet of the capillary to the wall of quartz cell. Since the tangential flow speed was much larger than that of the convection due to the 
(a)

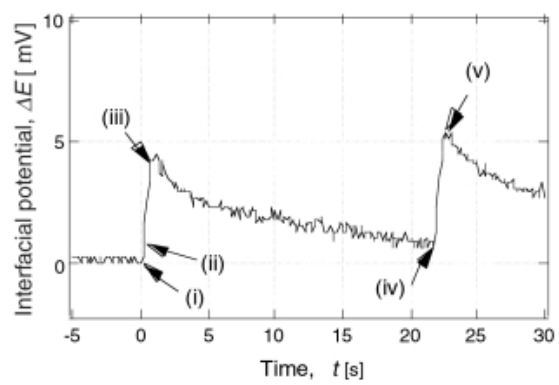

(b)

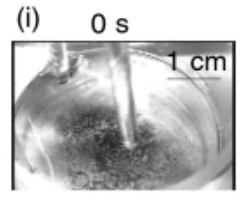

(iv) $21.0 \mathrm{~s}$

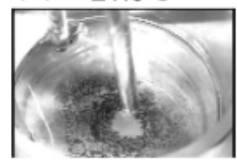

(ii) $0.3 \mathrm{~s}$

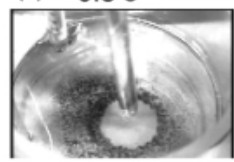

(v) $22.0 \mathrm{~s}$

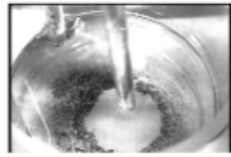

(iii) $0.8 \mathrm{~s}$

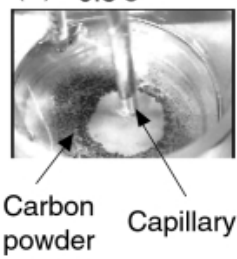

Fig. 4 Time course of the interfacial electrical potential, (a), and photo images of the interface, (b)-(i) - (v). The time at which the first oscillation started was set to zero. (b)-(i), (ii), and, (iii) indicate 0 , 0.3 , and $0.8 \mathrm{~s}$ since the first oscillation started. (b)-(iv) and (v) indicate 21.0 and $22.0 \mathrm{~s}$ after the first oscillation at which the second oscillation occurred. ${ }^{26}$

injection of surfactant solution, this phenomenon is specific to the oscillation. The capillary tip was very small in relationship to the inner diameter of the cell, and the concentration of $\mathrm{SC}_{\mathrm{n}} \mathrm{S}$ near the interface below the capillary was larger than that near the wall of the cell. As a result, the flow can be logically attributed to the Marangoni convection. This is likely to have been brought about by the generation of heterogeneity of interfacial tension due to locally adsorbed surfactant ions at the interface below the capillary, where the interfacial tension should be smaller than in the surrounding area. In most reports concerning the oscillatory phenomena at the liquid/liquid interfaces, chemical reactions at the interfaces were mainly discussed. Of course, the chemical reactions at the interface, including the adsorption or desorption processes, are very important elements in the oscillation, but our idea indicates that the complex reaction scheme is not always necessary for the oscillations.

Figure 4 shows the time course of the interfacial potential for $\mathrm{SC}_{6} \mathrm{~S}$ (in Fig. 4 (a)), and photos of the interface, (in Fig. 4 (b)-(i) to (v)). ${ }^{26}$ The color tone of these photos was modified to make the image clear. In the graph, time zero means the time at which the first abrupt increase of the potential occurred. The value of $\Delta E$ when the experiment started was set to zero, and the time at which the abrupt increase of the potential started was set to zero. The points corresponding to each photo, Fig. 4 (b)(i) to (b)-(v), are indicated in Fig. 4 (a). Figure 4 (b)-(i) is a photo of the start of an oscillation $(0 \mathrm{~s})$. Soon after that, a transparent region appeared at the liquid/liquid interface (Fig. 4 (b)-(ii), $0.3 \mathrm{~s}$ ). Within $1 \mathrm{~s}$, the region expanded fast, (Fig. 4 (b)(iii), $0.8 \mathrm{~s}$ ), and its size almost reached the maximum. After the oscillation, it gradually shrank, as shown in the photo (Fig. 4 (b)-(iv), $22 \mathrm{~s}$ ), which was taken just before the second

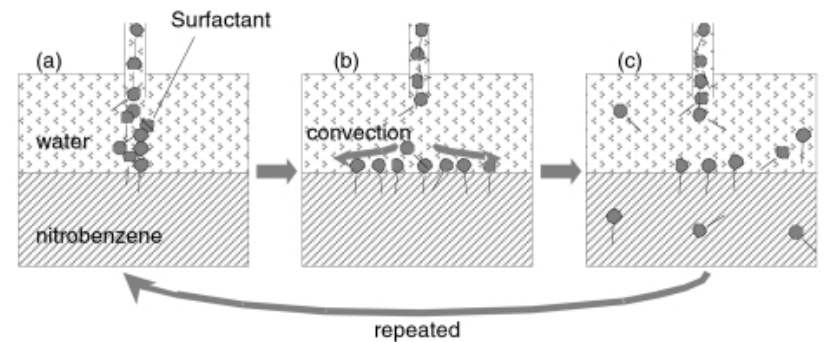

Fig. 5 The model of hydrodynamically induced chemical oscillation. Induction period, (a); generation and development of a Marangoni convection, (b); desorption process with decay of the Marangoni convection, (c). ${ }^{25}$

oscillation. As shown in Fig. 4 (b)-(v) (23 s), which was taken at the second oscillation, the transparent region expanded again. Comparing the time course of the interfacial potential with these photos, one could conclude that the surfactant ions adsorbed at the transparent region. From these results, the abrupt adsorption of surfactant ions at the interface could result from the adsorption of hexyl-sulfate ions that transferred with the flow along the interface. This flow is the Marangoni convection, and the apparent adsorption rate would reflect the magnitude of the convection.

From these results, we can propose the mechanism of the oscillation phenomena using Fig. 5. The origin of the abrupt adsorption of ions is almost the same for all materials. The capillary tip is very small in relationship to the inner diameter of the cell, and the concentration of surfactant ions near the interface below the capillary is larger than that near the wall of the cell. As a result, surfactant ions adsorb at the interface below the capillary more than the surrounding area (Fig. 5, (a)). Consequently, the Marangoni convection along the interface from the area below the capillary to the wall of the cell is brought about by the generation of the heterogeneity of interfacial tension. The convection can transfer the ions from the region below the capillary outlet toward the interface, which results in an abrupt adsorption of the surfactant ions at the interface (Fig. 5, (b)). The convection is suppressed soon due to the viscosity of liquid and reduction of heterogeneity of the interfacial tension, and the adsorption process enforced by the convection is damped. The concentration of surfactant ions in the bulk phase is small, except below the capillary; surfactant ions adsorbed at the interface are likely to desorb into the bulk (Fig. 5 (c)). Hence, desorption processes becomes dominant. However, some time later, the following oscillation can occur due to the ions being transferred by continuous injection. In Fig. 5, we mainly focus on the generation and reduction of the convection with the adsorption and desorption of ions, respectively, and do not pay much attention to the form of the ions which dissolve into the bulk phase from the interface. Precisely speaking, we must note that, although the surfactant ions dissolve into the aqueous phase as dissociated anions shown in Fig. 5, they dissolve into the nitrobenzene phase along with counter ions such as sodium ions. When nitrobenzene phase includes hydrophilic ions, dodecyl sulfate ions can dissolve as anions by the ion exchange process. Such examples will be discussed in the following section. In either case, as the form of ion is not directly related to the mechanism above, the counter ions are not shown in Fig. 5. The most important feature of the oscillation is that the convection can periodically grow and reduce.

We found that when a surfactant with larger surface activity 
(in this case, with larger carbon number, $n$ ) was used or when the surfactant solution was injected at a larger rate, the convection became large and explicit oscillatory patterns tended to disappear after abrupt adsorption. These results can be explained by postulating that the convection is large due to a large heterogeneity of interfacial tension and that adsorption of the convection continued and overcame the desorption process. The detailed results were discussed in our previous paper. ${ }^{25,26}$ On the other hand, when a surfactant solution with low concentration, $2 \mathrm{mM}$ SDS solution, was induced at a low rate with $2 \mu \mathrm{l} / \mathrm{min}$, we could not observe abrupt adsorption at all; in such cases the interfacial tension and potential changed gradually to the equilibrium state. This is because the heterogeneity of interfacial tension is not enough to generate a large convection.

\subsection{Ion exchange reaction}

In the sections above, we paid attention mainly to the abrupt ion adsorption process. In this section, we discuss the change of desorption process in the presence of hydrophilic ions in the oil phase that had the same electronic charge as the surfactant molecules. ${ }^{24}$ If these ions are present near the interfacial region, we could expect that an ion exchange between them and the charged surfactant molecules would occur and would accelerate the desorption of the surfactant molecules. Then we measured the chemical oscillation in the presence of a small amount of tetra- $n$-butylammonium halogenide $\left(\mathrm{TBA}^{+} \mathrm{X}^{-}\right)$that dissociates to the water-insoluble cation $\mathrm{TBA}^{+}$and hydrophilic anion $\mathrm{X}^{-}$in the oil phase. The concentration of these materials, $\mathrm{TBA}^{+} \mathrm{X}^{-}$, was $1.0 \times 10^{-4} \mathrm{M}$. $\mathrm{TBA}^{+}$was already present as a supporting electrolyte at $0.1 \mathrm{M}$, thus we could discuss the effect of the presence of the anions $\mathrm{X}^{-}$on the desorption process. The results are shown in Fig. 6 (a). The relaxation time was drastically accelerated by the presence of hydrophilic $\mathrm{X}^{-}$in the oil phase. As for all the experiments in the former sections, surfactant solutions were continuously injected for a few hours. On the other hand, in the experiments discussed here, we stopped the injection of the SDS solution soon after the abrupt adsorption of ions occurred, and hence, we did not see a periodic change of interfacial tension

Since the ion exchange should occur at the oil/water interface, we expected that the more the standard free energy of transfer of the anions from the interface to the water phase would decrease, the more efficiently the ion exchange that would occur between the hydrophilic anions and $\mathrm{DS}^{-}$ions, resulting in the acceleration of the desorption of the DS- ions from the interface. When the ion exchange occurs at the interface, the decrease of the standard free energy of the anions is the change of free energy of transfer from the interface to the water phase $\left(\Delta G_{\mathrm{i}-\mathrm{w}}\right)$. However, although water and nitrobenzene are immiscible, the interface between them should have some thickness where the water molecules and nitrobenzene molecules are mingled at the molecular level. Thus, we roughly estimated the average of the free energy of the anion at the interface $\left(G_{\mathrm{i}}\right)$ as the mean value of the free energy of the anion in the water phase $\left(G_{\mathrm{w}}\right)$ and that in the oil phase $\left(G_{0}\right)$, namely $G_{\mathrm{i}}=\left(G_{\mathrm{w}}+G_{\mathrm{o}}\right) / 2$. Then $\Delta G_{\mathrm{i}-\mathrm{w}}$ value was $G_{\mathrm{w}}-G_{\mathrm{i}}=\left(G_{\mathrm{w}}-G_{\mathrm{o}}\right) / 2=\Delta G_{0-\mathrm{w}} / 2$, where $\Delta G_{\text {o-w }}$ is the standard free energy of transfer from the oil phase to the water phase. This is a valid assumption for surface inactive ions such as halogenide ions. The $\Delta G_{\text {i-w }}\left(=\Delta G_{\text {o-w }} / 2\right)$ values of $\mathrm{Cl}^{-}, \mathrm{Br}^{-}$, and $\mathrm{I}^{-}$at the W/NB interface were $-15.3,-14.3$, and -9.4 $\mathrm{kJ} / \mathrm{mol}$, respectively, as calculated from $\Delta G_{\text {o-w }}$ values obtained by electrochemical measurements. ${ }^{44}$ The desorption rate of each case was determined by $1 / \Delta t$, where $\Delta t$ is the period for the interfacial tension to become the initial value again after the (a)

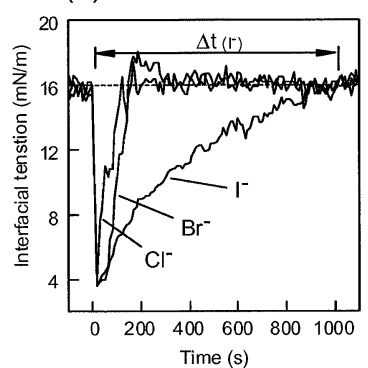

(b)

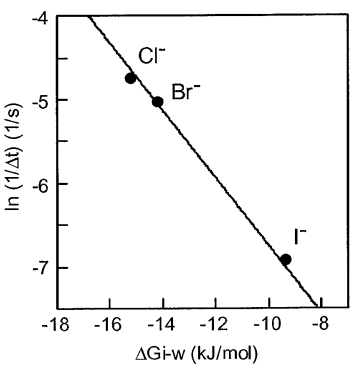

Fig. 6 (a) Time courses of the interfacial tension when hydrophilic anions $\left(\mathrm{Cl}^{-}, \mathrm{Br}^{-}\right.$and $\left.\mathrm{I}^{-}\right)$were in the oil phase. The concentration of these anions was $1.0 \times 10^{-4} \mathrm{M}$. (b) Dependence of the desorption rates $(\ln (1 / \Delta t))$ on the standard free energy of ion transfer from the W/NB interface to the water phase $\left(\Delta G_{\mathrm{i}-\mathrm{w}}=\Delta G_{\mathrm{o}-\mathrm{w}} / 2\right)$. The solid straight line with slope $-1 / R T\left(R\right.$ : gas constant $8.314 \mathrm{~J} \mathrm{~K}^{-1} \mathrm{~mol}^{-1}, T$ : temperature $298 \mathrm{~K}$ ) was obtained by the least square fitting method. ${ }^{24}$

oscillation. $\Delta t$ values of each ion $\left(\mathrm{Cl}^{-}, \mathrm{Br}^{-}\right.$, and $\left.\mathrm{I}^{-}\right)$were $110 \mathrm{~s}$, $150 \mathrm{~s}$, and $1000 \mathrm{~s}$, respectively. Figure 6 (b) shows the dependence of the desorption rate of the $\mathrm{DS}^{-}$ions on $\Delta G_{\mathrm{i}-\mathrm{w}}$ of $\mathrm{Cl}^{-}, \mathrm{Br}^{-}$, and $\mathrm{I}^{-}$from nitrobenzene to water. We can consider that the desorption rate is proportional to the exponential of $-\Delta G_{\mathrm{i}-\mathrm{w}} / R T$ ( $R$ : gas constant, $T$ : temperature). We plotted the dependence of the log value of the desorption rate $(\ln (1 / \Delta t))$ on the standard free energy of transfer from the interface to the water phase $\left(\Delta G_{\mathrm{i}-\mathrm{w}}\right)$. A solid straight line with the slope $-1 / R T$ ( $R$ : $8.314 \mathrm{~J} \mathrm{~K}^{-1} \mathrm{~mol}^{-1}, T: 298 \mathrm{~K}$ ) was obtained by the least square fitting method. The line fit the experimental results quite well. This result strongly supported the ion exchange model. Thus we could consider that the $\Delta G_{\mathrm{i}-\mathrm{w}}$ value of the coexisting ions in the oil phase strongly affected the desorption rate, namely the relaxation process in the chemical oscillation induced by the adsorption of charged surfactants.

Now let us return to Fig. 6 (a). The adsorption process was almost same for all the conditions. However, only the desorption processes were quite different from each other. The effects of the chemical reaction may explicitly appear due to the decay of the hydrodynamic effect during the desorption process.

\section{Conclusion}

We successfully observed the oscillations of interfacial tension and electrical potential during the adsorption and desorption processes of surface-active ions at the water/nitrobenzene interface using anionic surfactants, sodium-alkyl-sulfates. The oscillation pattern consisted of a repetition of abrupt adsorption and gradual desorption. Furthermore, we monitored the movement of the interface by taking photo-images with a CCD camera, and analyzed the relationship between the interfacial flow and the ion adsorption kinetics. Consequently, we could propose the mechanism of our chemical oscillation system, that is to say, the abrupt adsorption resulted from the generation of the Marangoni convection and the gradual desorption after the adsorption process was appeared after decay of the convection.

Chemical oscillations have often been discussed from the viewpoint of interfacial instability, which is caused by a fluctuation of the concentration of the adsorbed ion at the interface, that is, by the fluctuations of the interfacial tension. However, the origin of the fluctuation has not been made clear. In addition, how the fluctuation grows regularly and 
periodically seems to be an unsettled question. On the other hand, in our system, the instability was caused regularly because the surfactant ions were transferred toward the same point at the interface at a constant injection rate. This fact leads to the well-ordered oscillation structure.

We also found the wave motion at the interface travels faster than the convective mass transfer. This is an interesting result because such a phenomenon can be a sign of a nonlinear phenomenon. For example, seismologists can predict an eruption of a volcano through the observation of a shaking of the earth's surface, an earthquake. Therefore, the result above indicated that, even in a liquid/liquid interface in a small glass cell, we could detect the sign of the nonlinear phenomenon, an abrupt adsorption of ions. The relationship between mass flow and wave motion at the liquid interface was investigated by dual-beam QELS method, which allows us to measure simultaneously the interfacial tension at two different points within an interface. The detailed results will be published elsewhere.

Kovalchuk and co-workers reported ${ }^{29-32}$ the surface tension oscillation at the water/air interface. According to their experiments, an oscillation of the surface tension was observed in a variety of surfactants. Although the examined interface and materials in their experiments were different from those of ours, it is interesting that the observed phenomena were similar to our results and that the proposed mechanism was also discussed by the generation of Marangoni convection. Their results and our results imply that the oscillation phenomenon is not a specific feature of a few materials. If we compare the phenomena at the liquid/liquid interface to those at the liquid/air interface, we find that the former are much more complicated than the latter because ions or molecules from another side could exchange or react with each other at the liquid/liquid interface. Although, as an example, we could show the role of ion exchange reaction at the chemical oscillation, there is, of course, room for further investigation about the reaction rate or mass transfer processes during the oscillations.

\section{Acknowledgements}

The present research was supported by a Grant-in-Aid for Scientific Research (B) (No. 13129203) from the Ministry of Education, Science, Sports, and Culture of Japan.

\section{References}

1. I. Benjamin, J. Phys. Chem., 1991, 95, 6675.

2. T. Shioya, S. Nishizawa, T. Hayashita, and N. Teramae, J. Am. Chem. Soc., 1998, 120, 11534.

3. S. Ishizaka, S. Kinoshita, Y. Nishijima, and N. Kitamura, Anal. Chem., 2003, 75, 6035.

4. H. Watarai, S. Tsukahara, H. Nagatani, and A. Ohashi, Bull. Chem. Soc. Jpn., 2003, 76, 1471.

5. M. Dupeyrat and E. Nakache, Bioelectrochem. Bioenerget., 1978, 5, 134.

6. K. Yoshikawa and Y. Matsubara, J. Am. Chem. Soc., 1983, 105, 5967.

7. K. Toko, K. Yoshikawa, M. Tsukiji, M. Nosaka, and K. Yamafuji, Biophys. Chem., 1985, 22, 151.

8. K. Yoshikawa, M. Shoji, S. Nakata, and S. Maeda, Langmuir, 1988, 4, 759.

9. S. Kai, E. Ooishi, and M. Imasaki, J. Phys. Soc. Jpn., 1985, $54,1274$.
10. N. Magome and K. Yoshikawa, J. Phys. Chem., 1996, 100, 19102.

11. A. Shioi, Y. Sugiura, and R. Nagaoka, Langmuir, 2000, 16, 8383.

12. K. Yoshikawa and Y. Matsubara, Biophys. Chem., 1983, 17,183 .

13. K. Maeda, S. Kihara, M. Suzuki, and M. Matsui, J. Electroanal. Chem., 1990, 295, 183.

14. K. Maeda, S. Nagami, Y. Yoshida, H. Ohde, and S. Kihara, J. Electroanal. Chem., 2001, 496, 124.

15. S. Sha, P. J. Abatti, K. Iko, T. Nakamoto, and T. Moriizumi, Jpn. J. Appl. Phys., 1991, 30(8A), L1435.

16. H. Suzuki and T. Kawakubo, Biophys. Chem., 1992, 45 , 153.

17. K. Arai, S. Fukuyama, F. Kusu, and K. Takamura, Electrochim. Acta, 1995, 40, 2913.

18. S. Takahashi, I. Tsuyumoto, T. Kitamori, and T. Sawada, Electrochim. Acta, 1998, 44, 165.

19. T. Yoshidome, T. Higashi, M. Mitsushio, and S. Kamata, Chem. Lett., 1998, 27, 855.

20. A. Shioi, K. Katano, and Y. Onodera, J. Colloid Interf. Sci., 2003, 266, 415.

21. V. Pimienta, R. Etchenique, and T. Buhse, J. Phys. Chem. A, 2001, 105, 10037.

22. V. Pimienta, D. Lavabre, T. Buhse, and Jean-Claude Micheau, J. Phys. Chem. B, 2004, 108, 7331.

23. T. Takahashi, H. Yui, and T. Sawada, J. Phys. Chem. B, 2002, 106, 2314.

24. H. Yui, Y. Ikezoe, T. Takahashi, and T. Sawada, J. Phys. Chem. B, 2003, 107, 8433.

25. Y. Ikezoe, S. Ishizaki, T. Takahashi, H. Yui, M. Fujinami, and T. Sawada, J. Colloid Interf. Sci., 2004, 275, 298.

26. Y. Ikezoe, S. Ishizaki, H. Yui, M. Fujinami, and T. Sawada, Anal. Sci., 2004, 20, 435.

27. J. Srividhya and M. S. Gopinathan, J. Phys. Chem. B, 2003, 107, 1438.

28. M. Szpakowska, I Czaplicka, E. Plocharska-Jankowska, and O. B. Nagy, J. Colloid Interf. Sci., 2003, 261, 451.

29. V. I. Kovalchuk, H. Kamusewitz, D. Vollhardt, and N. M. Kovalchuk, Phys. Rev. E, 1999, 60, 2029.

30. N. M. Kovalchuk and D. Vollhardt, J. Phys. Chem. B, 2000, 104, 7987.

31. N. M. Kovalchuk, V. I. Kovalchuk, and D. Vollhardt, Phys. Rev. E, 2001, 63, 031604.

32. O. V. Grigorieva, N. M. Kovalchuk, D. O. Grigoriev, and D. Vollhardt, J. Colloid Interf. Sci., 2003, 261, 490.

33. References in Ref. 23.

34. H. Lamb, "Hydrodynamics", 6th ed., 1932, Cambridge University Press, London.

35. R. H. Kathyl and U. Ingard, Phys. Rev. Lett., 1967, 19, 64.

36. M. A. Bouchiat and J. Meunier, Phys. Rev. Lett., 1969, 23, 752.

37. D. Langevin and M. A. Bouchiat, J. Physique, 1972, 33, 101.

38. D. Langevin, J. Colloid Interf. Sci., 1981, 80, 1145.

39. D. Byrne and J. C. Earnshaw, J. Phys. D, 1979, 12, 2951.

40. A. Trojanek, P. Krtil, and Z. Samec, Electrochem. Commun., 2001, 3, 613.

41. Y. Uchiyama, I. Tsuyumoto, T. Kitamori, and T. Sawada, Langmuir, 2000, 16, 6597.

42. M. Takahashi, H. Yui, Y. Ikezoe, and T. Sawada, Chem. Phys. Lett., 2004, 390, 104.

43. T. Morisaku, H. Yui, M. Iwazumi, Y. Ikezoe, M. Fujinami, and T. Sawada, Anal. Chem., 2004, 76, 2314.

44. J. Koryta, Electrochim. Acta, 1984, 29, 445. 